\title{
Effects of Pedunculopontine Nucleus stimulation on human bladder function
}

\section{Abstract}

1) Aims: The pedunculopontine nucleus (PPN) is a deep brain stimulation target for Parkinson's disease (PD). Unilateral PPN stimulation has been described in a previous case report to provoke urinary frequency, urgency and detrusor overactivity, due to probable activation of the pontine micturition centre. Our aim was to evaluate the effect of bilateral PPN DBS on urodynamic parameters and to investigate the likely mechanisms using probabilistic tractography.

2) Methods: 6 male PD subjects with bilateral PPN deep brain stimulators were recruited. Urodynamic bladder filling assessments were carried out with the stimulators ON and OFF. 2 subjects also had diffusion-weighted and T1weighted MRI scans performed and probabilistic tractography was carried out to describe white matter connections with the stimulated area.

3) Results: 5 subjects completed urodynamic testing. PPN DBS did not give rise to detrusor overactivity or lower sensory thresholds during bladder filling. However, there was a significant increase in maximal bladder capacity with stimulation: mean bladder volume at maximal capacity was $199 \mathrm{mls}$ (range 103-440) ON stimulation compared with $131 \mathrm{mls}$ (range 39-230) OFF stimulation. Tractography demonstrated extensive connectivity to cortical and subcortical regions, some of which have been implicated in bladder control. Fibre pathways also passed close to the vicinity of the pontine micturition centre.

4) Conclusions: Bilateral PPN DBS did not have a detrimental effect on urodynamic filling parameters or produce detrusor overactivity, but did slightly increase maximal capacity. Possible mechanisms include long-range connectivity or local effects at the pontine micturition centre.

\section{$\underline{\text { Introduction }}$}

Deep brain stimulation (DBS) is well-established in the treatment of movement disorders such as Parkinson's disease and dystonia. Furthermore there is growing 
interest in its applications in other spheres including neuropsychiatry and autonomic regulation. Research on the use of DBS for urinary functional modulation is a result of clinical studies in which subjects with DBS for other indications, such as movement disorders or chronic pain, are willing to undergo urodynamic testing for the benefit of research. It has been demonstrated that DBS of the subthalamic nucleus (STN) typically increases bladder capacity and reduces detrusor overactivity ${ }^{12}$, while DBS of the thalamus significantly decreases bladder volume at 'first' and 'strong' desire to void and maximum bladder capacity ${ }^{3}$. DBS of the globus pallidus interna in patients with dystonia reduces urine flow rate, increases post-void residual and reduces detrusor overactivity ${ }^{4}$, and DBS of the periaqueductal grey area (PAG) increases maximal bladder capacity ${ }^{5}$. This growing literature provides valuable information about potential targets for functional manipulation of bladder function and outlines urinary side-effects from various targets. DBS-bladder studies are also an excellent supplement to research on the neural control of the bladder derived from other investigative modalities including functional MRI.

In a recent case report, a patient with Parkinson's disease (PD) experienced new onset lower urinary tract symptoms immediately after unilateral, right-sided DBS of the pedunculopontine nucleus (PPN), initially associated with stimulation of the most caudal contact, not having experienced urinary symptoms pre-operatively ${ }^{6}$. It was suggested that the detrusor overactivity, along with symptoms of urinary frequency, urgency and urge incontinence, occurred due to an effect of the stimulation on the pontine micturition centre (PMC) in the dorsolateral pons. The PPN is a diffuse cell group located in the rostral pontine/caudal mesencephalic tegmentum. It is part of the mesencephalic locomotor region and is known to undergo degeneration in $\mathrm{PD}^{7}$. In 
humans, it has been stimulated bilaterally, with varying results, for the treatment of severe gait disturbance in $\mathrm{PD}^{8}$.

In this study we were interested to discover, using urodynamics, whether a detrimental effect on bladder function occurs with bilateral stimulation of the PPN, (the usual mode of implantation ${ }^{8}$ ). The second aim was to understand the mechanism of any changes in urological function, particularly, whether any effects are mediated via surrounding areas such as the PMC and pontine storage centre (PSC); the PAG; or via distant nodes within the "central bladder network" - the complex network of sensory, motor and autonomic regions involved in bladder sensation, continence and voiding. We carried out urodynamics ON and OFF PPN DBS in a small series of subjects, and used diffusion tensor imaging (DTI) in two of these subjects to map the probabilistic white matter pathways connecting the DBS "volume of activated tissue" with the rest of the brain.

\section{Subjects and methods}

6 subjects with PD who had bilateral PPN DBS electrodes inserted between 2007 and 2011 participated in the study. The study was carried out in accordance with the Declaration of Helsinki and received approval from the Oxfordshire Research Ethics Committee B (study 09/H0605/62). 
Five procedures were performed in Oxford and one at the National Hospital for Neurology and Neurosurgery, London (subject 5). For the Oxford patients, the electrode trajectory was based on visual identification of landmarks on a pre-operative MRI; target was dorsolateral to the superior cerebellar peduncular decussation and medial to the medial lemniscus (figure 1). For further surgical details, see ${ }^{9}$. For patient 5, electrode placement was guided by stereotactic proton density MRI sequences that were also used to confirm accurate lead placement. For further details see ${ }^{6}$.

Imaging

Subjects underwent MRI on a 1.5 Tesla Phillips Achieva magnet shortly prior to DBS surgery. Diffusion-weighted data was acquired using a single-shot echo planar imaging sequence $(176 \times 176$ reconstructed matrix, voxel size $=1.8 \times 1.8 \times 2 \mathrm{~mm}$, repetition time $=9390 \mathrm{~ms}$, echo time $=65 \mathrm{~ms}$, and slice thickness, $2 \mathrm{~mm})$. DTI data were acquired with 33 directions, (b value $=1200 \mathrm{~s} / \mathrm{mm}^{2}$ ) and one image without diffusion weighting was obtained $(\mathrm{b}=0)$ ). A high-resolution T1-weighted structural image (voxel size $1 \mathrm{x} 1 \mathrm{x} 1 \mathrm{~mm}^{3}$ ) was acquired to check for gross structural abnormalities and used here for registration and structural analysis purposes, with a three-dimensional 'FLASH' sequence $\left(\mathrm{TR}=12 \mathrm{~ms}\right.$, $\mathrm{TE}=5.6 \mathrm{~ms}$, flip angle $=19^{\circ}$, with elliptical sampling of $k$-space, giving a voxel size of $1 \times 1 \times 1 \mathrm{~mm}^{3}$ in $5.05 \mathrm{~min}$ ). Post-operative CT scans were also obtained to determine electrode location. 
All patients had their PD medications withheld for 12 hours prior to testing, with the exception of patient 5 who took a quarter of the normal dose on the morning of testing.

Urodynamics were carried out in accordance with International Continence Society guidelines.

Participants were asked to void urine in order to fully empty their bladder prior to testing and urinalysis was performed to exclude a urinary tract infection. A dual lumen urethral catheter ( $8 \mathrm{Fr}$ ) was used to fill the bladder and measure intravesical pressure. A rectal catheter (4.5 Fr) was inserted to measure abdominal pressure and thus derive detrusor pressure by subtracting the abdominal pressure from the intravesical pressure.

The bladder was infused with a sterile solution of isotonic saline at an average rate of $30 \mathrm{ml} \mathrm{min}{ }^{-1}$. Urodynamic parameters were evaluated, including detrusor contraction amplitude and bladder volume at initial sensation of bladder filling, initial desire to void, normal desire to void, strong desire to void, and maximal capacity. When the patient reported being unable to tolerate further filling (i.e. maximal capacity), the infusion was stopped immediately. Bladder filling was carried out twice with PPN DBS turned 'ON' and twice with PPN DBS turned 'OFF'; the 'ON'/ 'OFF' order was pseudorandomised. The patient and clinician performing urodynamics were blinded to stimulation parameters. There was a 10 minute interval between changing the DBS 
stimulation setting and starting the next urodynamic cycle to ensure that the effects of stimulation being ON or OFF had reached a steady state.

Voiding parameters were not recorded due to difficulties in mobilising the patients to a voiding position for each of the ' $\mathrm{ON}$ ' and 'OFF' trials, given that they were in an "off medication" state, which exacerbated akinesia and tremor. We were also concerned that catheter displacement would occur while mobilising and felt that this would potentially jeopardise the filling part of the study if patients were not able to tolerate multiple catheterisations.

Questionnaires

One patient completed the King’s Health Questionnaire pre-operatively and 4 months post-operatively. The rest were recruited post-operatively, and there was therefore no opportunity to obtain pre-operative data on urinary symptoms in these patients.

Diffusion tensor image analysis

Two subjects underwent DTI analysis. Images were analysed using the FMRIB Software Library (FSL), Release 5.0 (C) 2012. A detailed description of analysis methods can be found in Fernandes et al. (2015) ${ }^{10}$. In summary, the post-operative CT was skull-stripped and used to locate the DBS electrode contacts. The postoperative CT and the DTI $b 0$ volume were co-registered to T1 space using the FLIRT tool (FMRIB, Oxford) and the resulting transformation matrix from DTI to T1 space 
was used to transform the DBS contact masks from T1 space to DTI space with a trilinear interpolation method. With the transformed electrode contact mask in DTI space, a mask of the voxel of maximum intensity was made. This mask was taken to represent the voxel within DTI space with the maximum probability of containing the electrode contact. The electric field distribution around the active electrode contacts (volume of activated tissue, (VAT)) was then modelled according to a previously described method ${ }^{10}$. To quantify and compare connectivity profiles and their relative strengths, the brain was parcellated into 116 cortical, subcortical and cerebellar regions based on the automated anatomical labelling (AAL) atlas ${ }^{11}$; each region represented a network node to which connectivity could be described.

The AAL template (originally in MNI space) was transformed into the DTI space of each individual using the existing T1 to DTI transformation matrix obtained in a previous step (see above), concatenated with the transformation matrix derived from linearly co-registering the ICBM152 in MNI space into the T1 space of the structural image with an affine registration (12 degrees of freedom) using a nearest neighbour interpolation. A separate PAG atlas ${ }^{12}$ was obtained and also transformed into the DTI space using the same method.

\section{Tractography}

The FDT toolbox (version 5.0, www.fmrib.ox.ac.uk.fsl) in FSL was used to process the diffusion data. Each subject's diffusion-weighted image was individually inspected for gross distortions, corrected for head motion and eddy currents and brain 
extracted. A Markov Chain Monte Carlo sampling algorithm was used to estimate the local probability of fibre direction in each voxel, using an automated estimation of two fibre directions within each voxel. Using the estimated VAT as a seed region, probabilistic tractography was applied at a single voxel level, sampling 5000 streamlines per voxel.

\section{Results}

\section{Subjects}

One subject was tested within a week of surgery and did not undergo the full cycle of 4 fills due to an operational failure with the urodynamics system. His data was therefore excluded from analysis. The remaining subjects $(n=5)$ were all male and the mean age was 62 years (range 54-71). Mean disease duration was 22.4 years (range 16-26) and the mean time since DBS implantation was 27 months (range 4-48). Stimulation parameters ranged from; amplitude 1.5 to $3.5 \mathrm{~V}$; pulse width 60 to $90 \mu \mathrm{s}$; and frequency 20 to $40 \mathrm{~Hz}$ (see table 1 for details). Electrode co-ordinates in MNI space are given in table 1.

\section{Urodynamics results}

There was a slight but insignificant increase in bladder volume at first desire to void with DBS 'ON' compared with 'OFF', (91.4 mls 'ON', (range 24-142); $53.8 \mathrm{mls}$ 'OFF' (range 22-87.5)). The mean bladder volume at maximal capacity was $199 \mathrm{mls}$ 
(range 103-440) ON stimulation compared with 131 mls (range 39-230) OFF. This effect was statistically significant ( $\mathrm{p}<0.05$, Wilcoxon test).

PPN DBS did not alter the amplitude or incidence of detrusor activity; only one subject displayed detrusor overactivity (patient 2) and this was present in all 4 filling trials, regardless of the DBS status. None of the other subjects had detrusor overactivity during any of the trials. (Figure 2, table 1).

\section{Questionnaire results}

Subject 2 completed the King's Health Questionnaire pre- and 4 months postimplantation. In this questionnaire, subjects rate how much symptoms affect them on a four point scale of "never”, “a little”, “moderately”, “a lot”. Pre-operatively, he reported experiencing frequency and nocturia “a lot”, urgency “moderately” and he “never” experienced urge, stress, or intercourse incontinence, nocturnal eneuresis, waterworks infections or bladder pain. Post-operatively, he experienced urinary frequency "never", nocturia and urgency "moderately" and, as pre-operatively, he “never” experienced urge, stress or intercourse incontinence, nocturnal eneuresis, infections or bladder pain.

Tractography results

Tractography demonstrated extensive connectivity between the VAT and cortical, subcortical and cerebellar regions for patients 2 and 4 (figure 3A,B). Connectivity common to both subjects included right sensorimotor cortex, bilateral thalamus, 
bilateral pallidum, bilateral cerebellum, cerebellar vermis and bilateral precuneus (table 2). There was no overlap between the paths and the outlined region of the PAG in either patient, although patient 2's tracts passed very close to the outer margins of the PAG (figure 3A,B). There is no validated atlas of the PMC or PSC available, however, both patients had significant projections through the pons. In particular, subject 4's tracts were positioned in the dorsolateral tegmentum close to where the PMC would be whereas projections from subject 2 were more extensive, covering ventral and dorsal regions (figure 3C).

\section{$\underline{\text { Discussion }}$}

The main purpose of this study was to investigate whether adverse effects of PPN stimulation on urinary control described in a case report ${ }^{6}$ are reproduced in patients with chronic, bilateral PPN DBS. Our primary conclusion is that in 5 chronically implanted subjects, PPN stimulation produced no significant worsening of urodynamic parameters. In particular there was no change in the incidence or amplitude of detrusor overactivity with DBS ON compared with OFF, and no significant reduction in bladder capacity or any other parameter.

We also observed a small increase in maximal bladder capacity with PPN DBS ON compared to OFF, thus we demonstrated an improvement rather than detrimental effects of DBS. There was a considerable spread in the magnitude of response to stimulation in terms of bladder capacity, across subjects. We could not attribute this to stimulation type (monopolar/bipolar), electrode location or duration of stimulation and suggest that further investigation is required to better understand this effect. 
In 2 subjects, probabilistic tractography from the VAT demonstrated long range connections to a number of regions that have been described as being linked with urinary storage or voiding, including the supplementary motor area (SMA), postcentral gyrus, superior frontal gyrus, thalamus, left putamen, pallidum, cerebellum and the precuneus, (the latter described in a connectivity-based analytic approach). Although further work is needed to understand the specific role of these areas in bladder control, it is thought that the role of the basal ganglia in the micturition cycle is largely inhibitory ${ }^{13}$, with activation of the pallidum during bladder filling in healthy subjects ${ }^{14}$ and activation of the putamen during bladder filling in subjects with PD ${ }^{15}$. The lentiform nucleus, which includes the pallidum, is also activated during urinary voiding implying a role in this motor process ${ }^{16}$. Regions within the frontal cortex, including the SMA and the superior frontal gyrus, are considered important for exercising inhibition of the micturition reflex, since lesions in these areas tend to produce detrusor overactivity ${ }^{13}$. The superior frontal gyrus has also been linked with decision-making and social and motivational aspects of bladder function ${ }^{17}$ and the SMA with motor control, being typically activated during voiding ${ }^{16}$ and pelvic floor relaxation/contraction ${ }^{18}$. The cerebellum is proposed to have a motor role during voiding ${ }^{16}$ and also to be involved in the processing of sensory information during bladder filling ${ }^{19}$. This is supported by evidence that cerebellar lesions have an effect on urinary storage and voiding processes. Michels et al (2014) ${ }^{17}$ found that the cerebellum was activated during interruption of micturition. The ventral posterior thalamic nucleus is involved in interoception ${ }^{20}$. Relatively little attention has been paid to the precuneus and its significance in micturition control, however its activation has been reported during void initiation ${ }^{17}$ and bladder distension ${ }^{21}$. 
Overall, our tractography data suggests connectivity between PPN and sensorimotor components of the bladder circuitry, which may contribute to the observed effects of stimulation. However, it is important to note that many established components of the bladder network, including the insula cortex and anterior cingulate cortex were not identified.

To provide more accurate tractography, it would be ideal to perform DWI scanning after implantation of the DBS lead, rather than pre-operatively and ideally at the time of urodynamics. This would ensure that any changes to white matter anatomy through disease progression or aging could be taken into account. This may be a possibility with forthcoming MRI compatible systems.

We used a PAG atlas to indicate the likely position of the PAG for each of the two subjects. We found that the white matter pathways did not pass through the PAG, including subject 4, for whom DBS had a larger proportional effect on bladder capacity. The PAG has been hypothesised to act as a "micturition switch" ${ }^{5}$ and modulation of activity within the PAG by PPN DBS could be hypothesised to alter micturition control but this is not supported by our data.

Finally, we examined the white matter projections through the pons. Although there is no validated human atlas of the PMC or PSC, it was clear that both subjects had projections through the pons, including the dorsolateral tegmentum close to where the PMC is located. The PMC contains several populations of neurons which project to the sacral parasympathetic nucleus of the spinal cord and Onuf's nucleus, and play a range of synchronized roles in the control of micturition including the disinhibition of 
spinal micturition-inhibitory pathways ${ }^{22}$. The PSC, ventrolateral to the PSC, also contains at least four neuronal populations with discharge patterns synchronous with the micturition cycle ${ }^{23}$. The PPN is located close to the locus coeruleus and the PMC to the extent that PMC neurons could be influenced by current spread from the DBS lead ${ }^{24}$. Based on our tractography it is plausible that PPN DBS could influence PMC activity via white matter projections. In contrast to the previous report of unilateral PPN DBS and its effects on the bladder, we describe the effect of bilateral PPN DBS. There is evidence that the pontine control of micturition may be right-lateralised ${ }^{25}$. The question of laterality may be addressed in future work.

The first major limitation of this study is the small number of subjects, making randomization difficult. PPN DBS is performed in relatively low volumes for PD compared with STN or GPi DBS, and the pool for recruitment is therefore very small. However, we believe that these findings are nonetheless important, particularly for neurosurgical teams who want to be more informed about possible side effects of PPN DBS. A second limitation is that that the results may have been influenced by a "carry over effect” of stimulation after turning the DBS OFF. However, this is unlikely, as generally the physiological effects of changing stimulation in the context of PD are manifested within seconds or minutes. Finally, we were not able to test voiding parameters in this study, an important area, given the possible influence of DBS on the PMC, and this should be investigated further. However, since the side-effect profile detailed in the Aviles-Olmos et al. (2011) ${ }^{6}$ case report concerned storage symptoms, we have investigated these in the first instance. 
It should be noted that the purpose of this study was not primarily to investigate whether PPN DBS might be a suitable target for neuromodulation to improve bladder symptoms. Less invasive treatment options, such as sacral neuromodulation or posterior tibial nerve stimulation are safer than DBS and have good efficacy in selected populations. Moreover, our results are specific to a small and biased cohort of patients, namely those with PD and predominant gait disturbance, thus selected for PPN DBS. The results should be interpreted within this context.

To summarise: we have studied the effect of PPN DBS on bladder filling parameters in 5 male subjects with PD. PPN DBS did not provoke detrusor overactivity or reduce sensory bladder filling thresholds. There was a small increase in maximal bladder capacity with PPN DBS ON, but larger studies are essential to validate this finding. Probabilistic tractography demonstrated a possible mechanism for the observed effects either via long-range connections to known cortical, subcortical and cerebellar regions for bladder control or via direct effects on the PMC.

\section{References}

1. Seif C, Herzog J, van der Horst C, Schrader B, Volkmann J, Deuschl G. et al. Effect of subthalamic deep brain stimulation on the function of the urinary bladder. Annals of neurology 55, 118-120 (2004).

2. Finazzi-Agro E, Peppe A, D'Amico A, Petta F, Mazzone P, Stanzione P. et al. Effects of subthalamic nucleus stimulation on urodynamic findings in patients with Parkinson's disease. The Journal of urology 169, 1388-1391 (2003).

3. Kessler TM, Burkhard FC, Z'Brun S, Stibal A, Studer UE, Hess CW. et al. Effect of thalamic deep brain stimulation on lower urinary tract function. European urology 53, 607-612 (2008).

4. Mordasini L, Kessler TM, Kiss B, Schupbach M, Pollo C \& Kaelin-Lang A. Bladder function in patients with dystonia undergoing deep brain stimulation. Parkinsonism \& related disorders 20, 1015-1017 (2014).

5. Green AL, Stone E, Sitsapesan HA, Turney BW, Coote JH, Aziz TZ et al. Switching off micturition using deep brain stimulation at midbrain sites. Annals of neurology 72, 144-147 (2012). 
6. Aviles-Olmos I, Foltynie T, Panicker J, Cowie D, Limousin P, Hariz M et al. Urinary incontinence following deep brain stimulation of the pedunculopontine nucleus. Acta neurochirurgica 153, 2357-2360 (2011).

7. Hirsch, E.C., Graybiel, A.M., Duyckaerts, C. \& Javoy-Agid, F. Neuronal loss in the pedunculopontine tegmental nucleus in Parkinson disease and in progressive supranuclear palsy. Proceedings of the National Academy of Sciences of the United States of America 84, 5976-5980 (1987).

8. Stefani, A. et al. Bilateral deep brain stimulation of the pedunculopontine and subthalamic nuclei in severe Parkinson's disease. Brain : a journal of neurology 130, 1596-1607 (2007).

9. Pereira, E.A., Muthusamy, K.A., De Pennington, N., Joint, C.A. \& Aziz, T.Z. Deep brain stimulation of the pedunculopontine nucleus in Parkinson's disease. Preliminary experience at Oxford. British journal of neurosurgery 22 Suppl 1, S41-44 (2008).

10. Fernandes HM, V.H.T., Boccard SG, Owen SLF, Cabral J, Drco G, Green AL, Fitzgerald JJ, Aziz TZ, Kringelbach ML Novel fingerprinting method characterises the necessary and sufficient structural connectivity from deep brain stimulation electrodes for a successful outcome. New Journal of Physics 17, 1-13 (2015)

11. Tzourio-Mazoyer N. Landeau B, Pappathanassiou D, Crivello F, Etard O, Delcroix N. et al. Automated anatomical labeling of activations in SPM using a macroscopic anatomical parcellation of the MNI MRI singlesubject brain. NeuroImage 15, 273-289 (2002).

12. Ezra, M., Faull, O.K., Jbabdi, S. \& Pattinson, K.T. Connectivity-based segmentation of the periaqueductal gray matter in human with brainstem optimized diffusion MRI. Human brain mapping 36, 3459-3471 (2015).

13. Sakakibara, R. Lower urinary tract dysfunction in patients with brain lesions. Handbook of clinical neurology 130, 269-287 (2015).

14. Nour, S., Svarer, C., Kristensen, J.K., Paulson, O.B. \& Law, I. Cerebral activation during micturition in normal men. Brain : a journal of neurology 123 ( Pt 4), 781-789 (2000).

15. Kitta T, Kakizaki H, Furuno T, Moriya K, Tanaka K, Shiga T. et al. Brain activation during detrusor overactivity in patients with Parkinson's disease: a positron emission tomography study. The Journal of urology 175, 994-998 (2006).

16. Shy M, Fung S, Boone TB, Karmonik C, Fletcher SG \& Khavari R. Functional magnetic resonance imaging during urodynamic testing identifies brain structures initiating micturition. The Journal of urology 192, 1149-1154 (2014).

17. Michels L, Blok BF, Gregorini F, Kurz M, Schurch B, Kessler TM et al. Supraspinal Control of Urine Storage and Micturition in Men-An fMRI Study. Cereb Cortex 25, 3369-80 (2014).

18. Seseke S, Baudewig J, Kallenberg K, Ringert RH, Seseke F \& Dechent P. Gender differences in voluntary micturition control: an fMRI study. NeuroImage 43, 183-191 (2008).

19. Athwal BS, Berkley KJ, Hussain I, Brennan A, Craggs M, Sakakibara R. et al. Brain responses to changes in bladder volume and urge to void in healthy men. Brain : a journal of neurology 124, 369-377 (2001). 
20. Bruggemann, J., Vahle-Hinz, C. \& Kniffki, K.D. Representation of the urinary bladder in the lateral thalamus of the cat. Journal of neurophysiology 70, 482-491 (1993).

21. Jarrahi B, Mantini D, Balsters JH, Michels L, Kessler TM, Mehnert U. et al. Differential functional brain network connectivity during visceral interoception as revealed by independent component analysis of fMRI TIME-series. Human brain mapping 36, 4438-4468 (2015).

22. Sasaki, M. Role of Barrington's nucleus in micturition. The Journal of comparative neurology 493, 21-26 (2005).

23. Sakakibara, R. et al. Firing patterns of micturition-related neurons in the pontine storage centre in cats. Autonomic neuroscience : basic \& clinical 99, 24-30 (2002).

24. Blanco, L., Ros, C.M., Tarragon, E., Fernandez-Villalba, E. \& Herrero, M.T. Functional role of Barrington's nucleus in the micturition reflex: relevance in the surgical treatment of Parkinson's disease. Neuroscience 266, 150-161 (2014).

25. Blok, B.F., Willemsen, A.T. \& Holstege, G. A PET study on brain control of micturition in humans. Brain : a journal of neurology 120 ( Pt 1), 111-121 (1997). 